HEALTHCARE DELIVERY

\title{
Description of an internal medicine outreach consultant appointment in western KwaZulu-Natal, South Africa, 2007 to mid-2014
}

\begin{abstract}
R I Caldwell, B Gaede, C Aldous
Doc Caldwell spent more than 25 years in private practice as a specialist physician in Pietermaritzburg, South Africa, and then took up a post at Grey's Hospital as outreach physician for internal medicine. He now does outreach on a sessional basis, and has a part-time position with the School of Clinical Medicine, University of KwaZulu-Natal, as undergraduate liaison between Pietermaritzburg and Durban. Bernhard Gaede was recently appointed Head of the Department of Family Medicine at UKZN. Prior to this he was Director of the Centre for Rural Health at UKZN for 4 years after working for more than a decade at Emmaus Hospital in the Drakensberg. Areas of interest and research have included the healthcare system, communitylevel care (including home-based care and traditional medicine), human rights and medical anthropology. Recent interests include health professional education and establishment of a decentralised teaching platform. Colleen Aldous is a senior lecturer in the School of Clinical Medicine at UKZN. She is a medical scientist with a PhD in science education and is involved in postgraduate research mentorship across several medical disciplines including surgery, orthopaedics, dermatology, paediatrics, opthalmology, general medicine and psychology. Her own research interest is human genetics, and she is a member of the national steering committee and working group reviewing National Department of Health policy guidelines for human genetics services.
\end{abstract}

Corresponding author: R I Caldwell (ric@caldwells.co.za)

This is a description of an internal medicine outreach appointment in western KwaZulu-Natal Province (KZN), South Africa (SA), from 2007 to mid-2014, facilitated by the transport services of the Red Cross Air Mercy Service (AMS) and funded by the KZN Department of Health. The hospital visits represented 'multifaceted' as opposed to 'simple' outreach. The AMS database of outreach visits was analysed according to frequencies of visits, number of patient contacts and number of contacts with medical personnel. A brief history of the outreach visits is given and their nature described. From January 2007 to the end of June 2014, the outreach physician undertook 481 hospital visits and visited seven hospitals (out of 21) more than 40 times each. A total of 3340 medical personnel contacts were made, and 5239 patients were seen. Other internal medicine specialists undertook an additional 199 visits, during which they made 1157 personnel contacts and saw 2020 patients. The combined total was therefore 680 visits undertaken, 4497 medical personnel contacts made and 7259 patients seen. The appointment of a dedicated outreach consultant for a particular discipline together with a reliable air and road transport system was successful in providing access to specialist care in rural settings. This strategy could be recommended throughout SA. Further studies would be required in order to assess outcomes.

S Afr Med J 2015;105(5):353-356. DOI:10.7196/SAMJ.9173

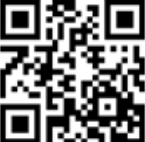

There is worldwide inequality between specialist services available to different sections of the population. This differential between urban and rural populations is starkly exemplified in KwaZulu-Natal Province (KZN), South Africa (SA). ${ }^{[1,2]}$ Outreach by specialists has been employed for years as a means of addressing equity of services. ${ }^{[3]}$ Such visits have been classified broadly as either simple or multifaceted outreach, with only the latter having had a significant impact on health outcomes. ${ }^{[3,4]}$ 'Multifaceted' visits represent heterogeneous activities and interactions between specialists and primary care practitioners that can include clinical service, in-service training for the staff, clinical audits or governance and even research. 'Simple' visits refer to mere displacement of an outpatient clinic to the visited site.

This is a description of an internal medicine outreach appointment in western KZN from 2007 to mid-2014, facilitated by the transport services of the Red Cross Air Mercy Service (AMS) and funded by the KZN Department of Health (KZN-DOH). The area covered by the outreach, the western half of the province, was designated as a pilot area for liaison between Grey's Hospital, Pietermaritzburg (PMB), and 21 district hospitals (Fig. 1). The population was approximately 3 million, most of whom relied on the public health service.

The outreach service was supported by the creation of full-time permanent posts for outreach for the major specialties by KZN-
DOH, based at Grey's Hospital, in 2006. A limited outreach service in the area had been in existence for more than 25 years, but the formal agreement in 1998 between KZN-DOH and the AMS put air transportation on a firm foundation, and made regular day visits to distant destinations possible.

In the PMB Department of Internal Medicine there were 14 consultants. The aim was that if each doctor 'adopted' a hospital to visit regularly, or if subspecialists rotated through different hospitals, the outreach physician, by undertaking frequent monthly visits, was able to ensure that all 21 hospitals received internal medicine outreach. Each hospital required a regular monthly visit from the major specialties, but with only one specialty per visit, so as not to dilute the personnel available to receive that visit. The visit was structured, with clinical and teaching rounds and a formal tutorial for all medical staff.

Arms-length-organised air and road transportation was the key to the outreach. The single turbo-prop Swiss Pilatus aeroplanes were robust and reliable, the pilots were numerous and competent, and the AMS was an experienced professional medical transport provider. Road transport was just as important, as statistics confirmed, and the AMS provided PMB with its own vehicle and driver.

Based on the experience gained by the hospital visits of the first author, a brief history of the outreach visits is given and their nature described. A data collection sheet, designed by internal medicine outreach, recorded visiting and visited personnel (doctors and medical students, 


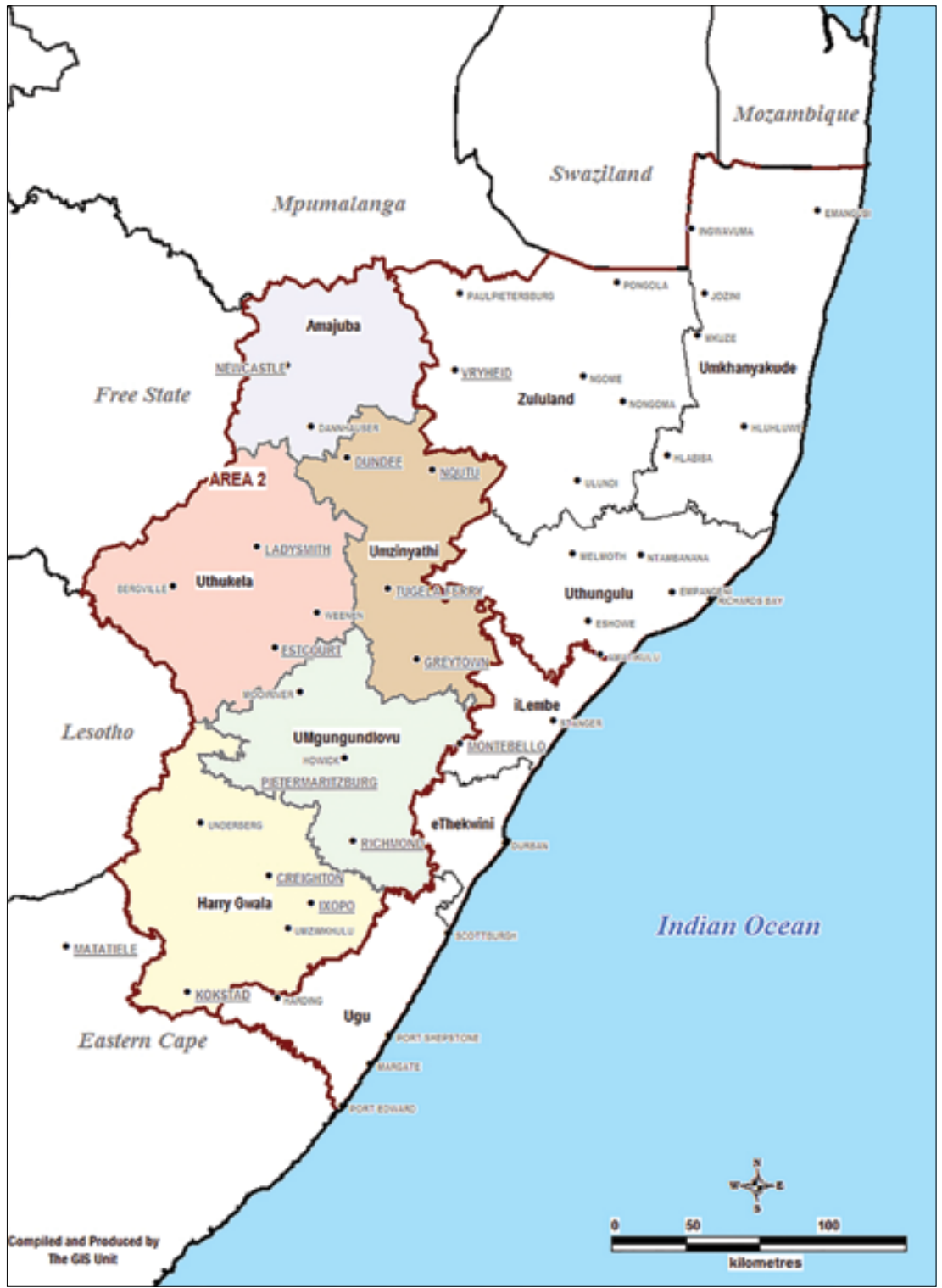

Fig. 1. KZN health districts, showing the Grey's Hospital catchment area.

the latter mainly on electives), patients seen, and their clinical details including the need for referral. The teaching session and problems encountered on the visit, and actions necessary, were also included. These forms were faxed to the AMS and filed in the Department of Internal Medicine at Grey's Hospital.

Visits were also recorded in a separate log, noting whether they were by air or road, as were cancellations, which occurred mainly due to bad weather. This log provided details on the number of visits per year and per hospital, and the number of flights v. road trips. AMS staff also maintained a $\log$ of all flights from their base in Durban, and of road trips done from PMB. As part of the review of the outreach service, the database of outreach visits from the AMS was analysed according to frequencies of visits, number of patient contacts and number of contacts with medical personnel. Permission for access to the database of the visits supported by AMS was obtained.

\section{Setting up the outreach programme}

During the setting up of the outreach service, all district hospitals in the catchment area of Grey's Hospital were visited by the outreach specialist in order to assess the needs, staffing situation and infrastructure. All hospitals were scheduled for regular visits by specialists, eight of which were allocated to the outreach specialist while 13 were distributed to other available consultants in the Department of Internal Medicine.

Visits were scheduled for particular days of the month: for example, Dundee Hospital was visited on the first Tuesday of every month. The same hospital would receive visits from consultants in other specialties on other regular days of the month as part of the AMS outreach programme. The doctors at remote peripheral hospitals could thus receive regular training from, and liaison with, different specialties.

In the planning of the hospital visit, various activities were identified as part of what the outreach visit should include. These components included an early-morning meeting, which incorporated a teaching session. This was to be followed by a booked outpatients clinic, where doctors from the local hospital and a nurse/interpreter were required to be present. A further component included a problem ward round of selected cases presented by the doctors concerned, where diagnosis, management and need for referral were to be covered.

\section{Description of the hospital visits \\ By air}

Outreach doctors awaited the AMS plane at PMB Airport at 07h30 from its Durban base. Arrival time at the remote hospitals depended on the flight plan. Flying time direct to Dundee, for instance, was 40 minutes, but it was much longer if the route was via Richards Bay and Ulundi to pick up and drop off other medical personnel. Road transport, provided by the relevant hospital, picked up the doctor at the airstrip for transfer. This journey might be short (for example, Dundee Hospital was very close to the airstrip), or longer (Charles Johnson Memorial Hospital was a 45-minute drive from the same airstrip). Arrival time at a hospital therefore ranged from $09 \mathrm{~h} 30$ to as late as $11 \mathrm{~h} 00$. Return flights from airstrips were usually from $15 \mathrm{~h} 30$ onwards, depending on the need to pick up other doctors before sunset, as many strips are very basic and do not have landing lights. Expected landing time at PMB Airport was therefore from $16 \mathrm{~h} 30$ onwards. Bad weather could result in the need to overfly to Durban, necessitating that personnel be driven back to PMB, which resulted in a very late but safe return.

\section{By road}

Road transport was necessary when there was no suitable airstrip for the hospital concerned, or if the distance from Grey's Hospital was short. Initially the AMS provided a vehicle for $\mathrm{PMB}$, with one of the doctors driving, but subsequently a permanent professional driver was employed. The vehicle left from Grey's Hospital, the departure time dependent on the required arrival time. For example, a 06h00 start was required to achieve an $08 \mathrm{~h} 00$ arrival at Church of Scotland Hospital (COSH), Tugela Ferry, in time for the teaching meeting. Return time varied according to the needs of the visit, usually by lunchtime with an $08 \mathrm{~h} 00$ arrival. The drop-off back at Grey's Hospital was therefore usually by $16 \mathrm{~h} 00$ or earlier. 
Table 1. Trips and hospital visits by the outreach physician year by year, January 2007 to end of June 2014

\begin{tabular}{lllll}
\hline Year & $\begin{array}{l}\text { Hospital } \\
\text { visits, } \boldsymbol{n}\end{array}$ & Flights, $\boldsymbol{n}$ & By road, $\boldsymbol{n}$ & Trips, $\boldsymbol{n}$ \\
\hline 2007 & 66 & 26 & 32 & 58 \\
2008 & 60 & 17 & 49 & 66 \\
2009 & 62 & 29 & 38 & 67 \\
2010 & 72 & 30 & 51 & 81 \\
2011 & 69 & 27 & 50 & 77 \\
2012 & 51 & 25 & 29 & 54 \\
2013 & 71 & 28 & 44 & 72 \\
2014 (to end June) & 30 & 13 & 21 & 34 \\
Totals & 481 & 195 & 314 & 509 \\
& & & &
\end{tabular}

\section{Hospital and other visits}

From January 2007 to the end of June 2014, the outreach physician undertook 481 hospital visits and made 509 trips. The discrepancy is explained by 41 Durban trips to attend KZN Pharmacotherapeutic Committee (KZN-PTC) meetings, by the fact that on occasion more than one hospital was visited per trip, and by the inclusion of occasional flights that had to be aborted prior to reaching the destination. Table 1 shows the yearly breakdown of such trips. The number of return flights (195) and road trips (314) is recorded.

\section{Frequency of visits per hospital}

Table 2 shows that with regular monthly visits, the outreach physician was able to visit four hospitals (out of 21) more than 50 times each, and a further three 40 or more times each.

\section{Multifaceted versus simple outreach}

The hospital visits represented multifaceted as opposed to simple outreach, and Table 3 shows the breakdown of personnel involved (doctors, medical students, nurses) and patients seen from 2007 to June 2014. A total of 3340 medical personnel contacts were made, and 5239 patients were seen.

\section{Involvement of the rest of the Department of Internal Medicine}

During this same period, other internal medicine specialists undertook 199 visits, giving a total of 680. A total of 4497 medical personnel contacts were made, and 7259 patients were seen. Table 4 details hospital visits by other physicians over this period.

\section{Discussion}

The internal medicine outreach programme in Area 2 of KZN had a large quantitative effect on access to specialist care. In 7.5 years the outreach physician undertook nearly 500 hospital visits, including almost 200 flights and more than 300 road trips, and undertook more than 3000 medical personnel contacts and more than 5000 patient consultations. Other specialists in the department provided a further 199 visits and saw 2020 patients. Much of the total output could therefore be attributed to the appointment of a dedicated outreach co-ordinator.

The PMB Department of Internal Medicine was fortunate in having a large enough consultant complement to allow the outreach physician to focus on this field rather than to be involved in clinical work at the tertiary hospital. Nevertheless, the appointment was a full-time one, requiring contribution to the running and welfare of the department.

\begin{tabular}{lll}
$\begin{array}{l}\text { Table 2. Number of visits by the outreach physician per hospital, } \\
\text { January 2007 to end June 2014 }\end{array}$ \\
\hline Order & Hospital & Visits, $\boldsymbol{n}$ \\
\hline 1 & COSH (Tugela Ferry) & 73 \\
2 & Vryheid & 60 \\
3 & Dundee & 53 \\
4 & Charles Johnson Memorial & 53 \\
& (Nqutu) & \\
5 & Estcourt & 47 \\
6 & Emmaus & 47 \\
7 & Rietvlei & 46 \\
8 & Christ the King (Ixopo) & 21 \\
9 & Greytown & 17 \\
10 & Richmond & 13 \\
11 & Madadeni (Newcastle) & 13 \\
12 & St Apollinaris (Creighton) & 7 \\
13 & Newcastle & 5 \\
14 & East Griqualand \& Usher & 5 \\
& (Kokstad) & \\
15 & Fort Napier (PMB) & 4 \\
16 & Ladysmith & 4 \\
17 & Town Hill (PMB) & 3 \\
18 & Tayler Bequest (Matatiele) & 3 \\
19 & Montebello & 3 \\
20 & Appelsbosch & \\
Total & Niemeyer (Utrecht) & \\
& & \\
& & \\
& &
\end{tabular}

Table 3. Visits, personnel contacts and patients seen, January 2007 to end June 2014

\begin{tabular}{lllll}
\hline Order & Hospital & $\begin{array}{l}\text { Visits, } \\
\boldsymbol{n}\end{array}$ & $\begin{array}{l}\text { Personnel } \\
\text { contacts, } \boldsymbol{n}\end{array}$ & $\begin{array}{l}\text { Patients } \\
\text { seen, } \boldsymbol{n}\end{array}$ \\
\hline 1 & COSH & 73 & 892 & 605 \\
2 & Vryheid & 60 & 247 & 1102 \\
3 & Dundee & 53 & 283 & 676 \\
4 & Charles Johnson & 53 & 279 & 444 \\
& Memorial & & & \\
5 & Estcourt & 47 & 385 & 320 \\
6 & Emmaus & 47 & 336 & 552 \\
7 & Rietvlei & 46 & 295 & 498 \\
8 & Christ the King & 21 & 242 & 602 \\
9 & Greytown & 17 & 158 & 276 \\
10 & Richmond & 13 & 66 & 24 \\
11 & Madadeni & 13 & 55 & 55 \\
$12-21$ & Others (<10 visits each) & 38 & 102 & 85 \\
Totals & & 481 & 3340 & 5239 \\
& & & &
\end{tabular}

The outreach physician was therefore on the call roster, did ward rounds at Edendale Hospital, a PMB regional/district hospital, initiated a telemetry link to peripheral hospitals, and became co-ordinator of 
Table 4. Other physician visits, 2007 to mid-2014

\begin{tabular}{llll}
\hline Hospital & Visits, $\boldsymbol{n}$ & Personnel contacts, $\boldsymbol{n}$ & Patients seen, $\boldsymbol{n}$ \\
\hline Vryheid & 1 & 3 & 25 \\
Dundee & 6 & 26 & 68 \\
Charles Johnson Memorial & 7 & 24 & 100 \\
Estcourt & 10 & 68 & 40 \\
Emmaus & 4 & 13 & 51 \\
Rietvlei & 16 & 63 & 121 \\
Greytown & 27 & 197 & 328 \\
Richmond & 3 & 20 & 7 \\
Madadeni & 24 & 176 & 174 \\
St Apollinaris & 2 & 8 & 28 \\
East Griqualand \& Usher & 53 & 296 & 748 \\
Ladysmith & 1 & 3 & 4 \\
Tayler Bequest & 9 & 69 & 61 \\
Appelsbosch & 36 & 191 & 265 \\
Totals & 199 & 1157 & 2020 \\
& & &
\end{tabular}

the elective medical student programme for internal medicine, such students often attending outreach visits. The incumbent was appointed as the outreach representative, for all specialties, on the KZN-PTC in Durban and organised two internal medicine weekend mini-symposia, held at Grey's Hospital in 2009 and 2011.

The very existence of an outreach programme presupposed an efficient, independent transport system, such as that provided by the AMS. The variable reliability of transport provided by recipient hospitals between hospital and airstrip emphasised this. It was air transport that made outreach feasible when a large geographical area was involved, scattered with numerous hospitals. This was of course the case with the catchment area of Grey's Hospital, and indeed for the whole of KZN. Air travel was more effective for the overall outreach service, as it could transport eight to nine individuals per flight, provide several pick-up and drop-off points, and therefore serve several hospitals on one round trip. There were two planes available on most travel days. However, flight visits were inefficient per individual specialist/ hospital, as timing of arrivals and returns was unpredictable, exacerbated by occasional bad weather.

The importance of safe road transport by the transport provider cannot be overstated. (The outreach physician undertook more road than air trips, and for him this was a more efficient means of transport.) Road travel usually implied a single destination and fewer passengers, a disadvantage for overall hospital provision. However, it allowed a predictable early start and arrival time, and return as soon as the visit was completed. Nevertheless, road visits could involve long and potentially dangerous journeys, with less capacity than journeys by air. hospital, the nature of the visit largely depending on the requirements, management and culture of the individual hospital. Some hospitals provided a large personnel contact and few patients, some the opposite, and some in between.

Simple outreach was never employed, and the comparison between Vryheid Hospital and $\mathrm{COSH}$ visits illustrated how variable the multifaceted category could be: ${ }^{[3,4]}$ at Vryheid there were few medical personnel and very many patients per visit, while at $\mathrm{COSH}$ many personnel and relatively few patients were encountered. At Vryheid there was a great need for a booked specialist outpatient clinic, to obviate or to facilitate referral. COSH had three senior medical officers with more than 65 years' continuous combined service, stability regarding medical managers and therefore less reliance on visiting consultants. There were many doctors at the teaching meeting, and a smaller patient load on problem ward rounds.

Limitations within the outreach programme included cancellation of visits, bad weather being the most common cause; inefficient transport from airstrip to hospital and vice versa; a relatively short time at the facility compared with the time taken for the travelling itself; ill-preparedness on the part of the recipient hospital; and lack of availability of some consultants, although the majority did participate.

Occasionally the outreach physician undertook 2- or 3-day trips during which he visited several hospitals and stayed overnight at bed-and-breakfast accommodation organised
The hospital visit varied from hospital to by the AMS. These road trips maximised time at a facility and reduced time spent travelling. However, comparative costs had to be considered, given that AMS provided an air service for multiple personnel' with built-in overheads. Moreover, not many consultants would find it acceptable, or even feasible, to spend longer periods away from their home base.

The considerable output achieved by the appointment of an outreach physician would on its own be a recommendation for this strategy throughout SA. ${ }^{[3,4]}$ How much this improved quality of care and outcomes was more difficult to determine, when an intervention was only on a monthly basis. More statistics would be required, preferably on a prospective basis, to enable outcomes to be compared and the effect of referrals and follow-up monitored. ${ }^{[5]}$

Reducing unnecessary use of services and influencing referral patterns favourably were clear aims of the outreach. Patients seen by the outreach specialist were those who might otherwise have required referral to the relevant regional or tertiary hospital. Only a minority of patients seen were referred, indicating that the intervention itself obviated the need for referral. ${ }^{[6]}$ The specialist could also expedite any future appointment.

Enabling peripheral hospital doctors to liaise with an individual specialist personally, rather than deal with an impersonal voice on the phone, encouraged them to make contact with the outreach physician on a regular, sometimes frequent, basis. ${ }^{[4]}$ More needed to be done in terms of establishing and enforcing protocols, employing morbidity-mortality meetings, etc. in order to make care more efficient. ${ }^{[4]}$

\section{Conclusion}

This study showed that the appointment of a dedicated outreach consultant for a particular discipline, together with a capable transport system, was successful in providing access to specialist care. This strategy could be recommended throughout SA. However, further studies, preferably prospective, would be required in order to assess outcomes and improvements in quality of care..$^{[4,5]}$

\footnotetext{
1. Tudor Hart J. 'The inverse care law'. Lancet 1971;1(7696):405-412. 2. Clarke DL, Aldous C. Surgical outreach in rural South Africa: Are we managing to impart surgical skills? S Afr Med 2014;104(1):57-60. [http://dx.doi.org/10.7196/SAMJ.7252]

3. Gruen RL, Weeramanthri TS, Knight SE, Bailie RS. Specialist outreach clinics in primary care and rural hospital settings. Cochrane Database Syst Rev 2004;(1):CD003798. [http://dx.doi. org/10.1002/14651858.CD003798.pub2]

4. Gaede B, McKerrow NH. Outreach programme: Consultant visits to rural hospitals. CME 2011;29(2):57-58.

5. Gruen RL, Weeramanthri TS, Bailie RS. Outreach and improved access to specialist services for indigenous people in remote Australia: The requirements for sustainability. J Epidemiol Community Health 2002;56(7):517-521. [http://dx.doi.org/10.1136/jech.56.7.517] 6. Gruen RL, Bailie RS, Wang Z, Heard S, O'Rourke IC. Specialist 6. Gruen RL, Bailie RS, Wang Z, Heard S, O'Rourke IC. Specialist
outreach to isolated and disadvantaged communities: A outreach to isolated and disadvantaged communities: A
population based study. Lancet 2006;368(9530):130-138. [http:// dx.doi.org/10.1016/ S0140-6736(06)68812-0]
}

Accepted 24 November 2014 